Dom. Cien., ISSN: 2477-8818

Vol. 4, núm. 1, enero, 2018, pp. 447-464

La gestión de las políticas públicas en las universidades: una aproximación a su caracterización en el Ecuador

\title{
La gestión de las políticas públicas en las universidades: una aproximación a su caracterización en el Ecuador
}

\author{
The management of public policies in universities: an approach to their \\ characterization in Ecuador
}

A gestão das políticas públicas nas universidades: uma abordagem à sua caracterização no Equador

\author{
Martha Cecilia-López ${ }^{\mathrm{I}}$ \\ cecilia.lopez@utelvt.ec \\ Luz M. Cifuentes-Quiñonez ${ }^{\text {II }}$ \\ luzmcifuentes@hotmail.com \\ Ana M. Palma-Avellán III \\ palmaavellan@gmail.com
}

Recibido: 7 de octubre de 2017 * Corregido: 28 de noviembre de 2017 * Aceptado: 10 de diciembre de 2017

I. Magister en Administración de Empresas, Licenciada en Ciencias de la Educación en la Especialización de Administración y Supervisión Educativa, Docente de la Universidad Técnica Luis Vargas Torres de Esmeraldas, Extensión la Concordia, Ecuador.

II. Magister en Docencia Mención Gestión en Desarrollo del Currículo, Magister en Administración de Empresas, Licenciado en Contabilidad y Auditoría, Doctora en Contabilidad y Auditoría, Técnica en Comunicación Radiofónica Para el Desarrollo, Docente en la Universidad Técnica Luis Vargas Torres De Esmeraldas, Esmeraldas, Ecuador.

III. Magister en Docencia Universitaria e Investigación Educativa, Diploma Superior en Educación Universitaria por Competencias, Diploma Superior en Investigación Científica, Ingeniero Industrial, Docente en la Universidad Laica "Eloy Alfaro” de Manabí, Manta, Ecuador. 


\title{
Resumen
}

Las políticas públicas son aquellas actividades de las instituciones gubernamentales, que van dirigidas a tener una influencia determinada sobre la vida de los ciudadanos, las cuales tienen que haber sido generadas o acompañadas en el marco de los procedimientos, instituciones y organizaciones gubernamentales; siempre se dirige a una situación que requiere una intervención por parte del Estado, para lograr ciertas transferencias directas o indirectas a los diferentes actores de la sociedad, y sobre todo, es la manera en que el Estado busca hacer efectivos los derechos que ha reconocido a sus ciudadanos. Estas decisiones gubernamentales, constituyen un importante aspecto de la realidad política de la sociedad y son objeto de muchos estudios desde la disciplina de ciencias políticas, y que, sin embargo, otras disciplinas como las ciencias gerenciales, la han abordado desde su perspectiva. La universidad como ente, no escapa de este proceso de generación de políticas públicas; no solo que los resultados de su gestión influyen en el desarrollo de la sociedad, sino que la misma gestión es producto de la interacción con las diferentes partes interesadas, sociedad, gobierno, profesores, empresas, entre otros. Aunque pareciera que el Estado y la Universidad están controlando el proceso de definición de los ejes centrales que dominan la gestión pública, la complejidad en la vida social y política actual, evidenciada en la heterogeneidad de los grupos y sectores que demandan acciones al Estado y a la Universidad, y en la cantidad de variables que intervienen en los problemas centrales de la sociedad, hacen que el desarrollo de políticas públicas sea una función clave para el sector universitario. Así, el esfuerzo de hacer política pública en la universidad, exige diseñar una hoja de ruta, que permita recorrer un camino desde lo que se tiene hasta el deber ser. En este artículo se plantea caracterizar las políticas públicas del sector universitario, como proceso de desarrollo de la región, mediante la revisión de literatura del tema. Se busca identificar cuáles son las semejanzas y diferencias con el resto de los sectores públicos, así como los aspectos claves de su gestión actual. Se pretende que sirva de referencia para trabajos posteriores.

Palabras clave: políticas públicas; educación superior; universidad.

\begin{abstract}
Public policies are those activities of government institutions, which are aimed at having a certain influence on the life of citizens, which must have been generated or accompanied within the
\end{abstract}


framework of procedures, institutions and governmental organizations; always addresses a situation that requires intervention by the State, to achieve certain direct or indirect transfers to the different actors of society, and above all, is the way in which the State seeks to make effective the rights it has recognized. its citizens. These governmental decisions are an important aspect of the political reality of society and are the subject of many studies from the discipline of political science, and that, however, other disciplines such as management sciences, have addressed from their perspective. The university as an entity does not escape from this process of generating public policies; not only that the results of its management influence the development of society, but that management itself is the product of interaction with different stakeholders, society, government, teachers, companies, among others. Although it seems that the State and the University are controlling the process of defining the central axes that dominate public management, the complexity of current social and political life, evidenced by the heterogeneity of the groups and sectors that demand actions from the State and the University, and in the number of variables that intervene in the central problems of society, make the development of public policies a key function for the university sector. Thus, the effort to make public policy in the university, requires designing a road map, which allows you to travel a path from what you have to what you should be. In this article, it is proposed to characterize the public policies of the university sector, as a process of development of the region, by reviewing literature on the subject. It seeks to identify what are the similarities and differences with the rest of the public sectors, as well as the key aspects of their current management. It is intended to serve as a reference for later works.

Keywords: public policies; higher education; college.

\section{Resumo}

As políticas públicas são as atividades das instituições governamentais, que visam ter uma certa influência na vida dos cidadãos, que deve ter sido gerada ou acompanhada no âmbito de procedimentos, instituições e organizações governamentais; sempre aborda uma situação que requer intervenção do Estado, para conseguir certas transferências diretas ou indiretas para os diferentes atores da sociedade e, acima de tudo, é a maneira pela qual o Estado busca efetivar os direitos reconhecidos. são cidadãos. Essas decisões governamentais são um aspecto importante da realidade política da sociedade e são objeto de muitos estudos da disciplina da ciência política e, no entanto, outras disciplinas como as ciências da gestão abordaram a partir de suas perspectivas. A universidade como entidade não escapa desse processo de geração de políticas públicas; não só que os resultados 
de sua gestão influenciam o desenvolvimento da sociedade, mas a própria gestão é o produto da interação com diferentes partes interessadas, sociedade, governo, professores, empresas, entre outros. Embora pareça que o Estado e a Universidade estão controlando o processo de definição dos eixos centrais que dominam a gestão pública, a complexidade da vida social e política atual, evidenciada pela heterogeneidade dos grupos e setores que exigem ações do Estado e da Universidade, e no número de variáveis que intervêm nos problemas centrais da sociedade, tornam o desenvolvimento das políticas públicas uma função fundamental para o setor universitário. Assim, o esforço para tornar a política pública na universidade, exige a concepção de um mapa rodoviário, que permite que você percorra um caminho do que você tem para o que deveria ser. Neste artigo, propõe-se caracterizar as políticas públicas do setor universitário, como um processo de desenvolvimento da região, revisando a literatura sobre o assunto. Ele procura identificar quais são as semelhanças e diferenças com o resto dos setores públicos, bem como os principais aspectos da sua gestão atual. Destina-se a servir de referência para trabalhos posteriores.

Palavras chave: políticas públicas; Educação superior; universidade.

\section{Introducción}

Las múltiples interacciones sociales, los cambios en los sistemas políticos, la producción de redes a escalas globales, nacionales y locales, la globalización, los reclamos de participación de la ciudadanía, los nuevos instrumentos de control de políticas públicas realizadas por el gobierno, la democratización política, entre otros factores, han generado un nuevo escenario para llevar a cabo el arte de gobernar (Castillo, 2017); en este contexto, la formulación de políticas públicas, toma un rol determinante en el quehacer político y de participación ciudadana.

Las políticas públicas se relacionan con el conjunto de instituciones que toman decisiones para resolver problemas y desajustes sociales, y se convirtieron en un agente que vino a permear la política en todas sus dimensiones y, con ello, a agregarse como tema de estudio de la ciencia política (Valencia \& Álvarez, 2008).

La política pública se trata principalmente de decidir quién recibe qué y quién paga; los dilemas éticos en las políticas públicas surgen por dos razones: los recursos son inadecuados para satisfacer todas las demandas, y las personas se ven obligadas a expresar sus valores e ideas sobre cómo hacer las cosas correctas (Bromell, 2012); es así como, el ciudadano común, que había delegado sus decisiones a los 
políticos, opta por reclamar sus espacios de participación, creando una nueva dinámica en los diferentes tribunas políticas.

En esta transformación, ya no es suficiente la importancia que se le ha dado al tema de políticas públicas, sino que ya se comienza a hablar de políticas públicas excelentes, políticas públicas de calidad, entre otros adjetivos que evidencia la importancia de este instrumento como transformador de la dinámica social. Así, una política pública de excelencia corresponde a aquellos cursos de acción y flujos de información relacionados con un objetivo político definido en forma democrática; los que son desarrollados por el sector público y, frecuentemente, con la participación de la comunidad y el sector privado; una política pública de calidad incluirá orientaciones o contenidos, instrumentos o mecanismos, definiciones o modificaciones institucionales, y la previsión de sus resultados (Lahera, 2004).

El reconocimiento al rol que juega el debate en el proceso de política pública, lleva a comprender que los actores participan en él, con las argumentaciones que mejor expresan los intereses que ellos promueven, que el debate es evolutivo, que se clausurará con la decisión de política pública y que esta recogerá aquel o aquellos argumentos aún vigentes que más se aproximen a los intereses que promueven los actores protagónicos del proceso y/o los llamados a decidir (Olavarría, 2007).

En cuanto al impacto en el quehacer universitario, la transformación de la educación en espacio público, la hace idónea para la intervención de las políticas públicas y de las autoridades públicas a través de un conjunto de iniciativas e instrumentos. El hecho de que la educación se haya convertido en un campo de intervención de las políticas públicas es el resultado de un largo proceso histórico que combina modernización y secularización. Esto que aparece como una característica propia de una sociedad moderna, es el resultado de procesos sociales complejos y de luchas sociales y políticas (Arcos, 2008).

Desde otro punto del escenario público, particularmente desde las oficinas de organismos internacionales, de gobiernos y partidos políticos, se reconoce la importancia de la educación superior como palanca de desarrollo, como una inversión más que un gasto, como un mecanismo de justicia social, a la vez que se reconocen los limitados recursos públicos para fortalecer la oferta en el sector, 
y se incrementan los mecanismos de supervisión, de control y evaluación de sus resultados, de sus insumos y de sus procesos (Acosta, 2013).

\section{Políticas públicas: una aproximación a su definición}

Las políticas públicas son un objeto de estudio complejo, lo que se refleja en la dificultad de encontrar una definición homogénea; la misma, debe comprenderse como el resultado de una acción colectiva que se desarrolla en lo público y de una serie de transacciones políticas, en donde el gobierno ya no tiene como único objetivo ejecutar lo planeado, sino también garantizar la coordinación y la cooperación de los actores clave (Torres-Melo \& Santander, 2013).

Cuando se habla de política pública se hace referencia a los procesos, decisiones, resultados, pero sin excluir conflictos entre intereses, tensiones entre diferentes definiciones del problema a resolver, entre diferentes racionalidades organizativas y de acción y entre diferentes perspectivas evaluadoras; se está ante un panorama lleno de poderes en conflicto, enfrentándose y colaborando ante opiniones y cursos de acción específicos (Aguilar \& Lima, 2009).

De acuerdo con Lupi, Rossi \& Mafullo (2014), las políticas públicas se definen como "procesos y campo de incidencia de la acción colectiva para la distribución de recursos, donde confluyen relaciones de actores sociales"; no son estáticas, evolucionan en el tiempo, y no siempre con un rumbo lineal ni previsible.

Por su parte Olavarría (2007), define una política pública como una intervención del Estado, expresada en una decisión o conjunto de decisiones de una autoridad pública, que considera un análisis técnico - racional para un tema determinado y una finalidad específica, que sigue un procedimiento formal, todo lo cual se da en el contexto de un intenso proceso político de confrontación y articulación de intereses.

Las políticas públicas abordan problemas socialmente tematizados (Lupi, Rossi \& Mafullo, 2014). Así, como construcción social, la política pública puede ser definida como una estrategia con la cual el gobierno coordina y articula el comportamiento de los actores a través de un conjunto de sucesivas acciones intencionales, que representan la realización concreta de decisiones en torno a uno o varios 
objetivos colectivos, considerados necesarios o deseables en la medida en que hacen frente a situaciones socialmente relevantes (Torres-Melo \& Santander, 2013).

La política pública presupone la tenencia de un problema común y de atención a todo; traducir un conjunto de visiones sobre el problema, alternativas de cómo enfrentarlo, gestionarlo, ejecutarlo, implica definir soluciones dentro del Estado para dar soluciones a temas prioritarios y el diseño de planes de acciones concretas de la asignación de recursos; debe contemplar acciones concretas, específicas (Salazar, 2007).

Como lo resume Arcos (2008), una política pública tiene como características: un contenido que expresa metas y resultados sustentados en una explicación sustantiva sobre la interacción de los factores que subyacen a los cambios sociales e institucionales; un programa de acción, lo que implica decisiones en torno a prioridades, líneas de acción, recursos, tiempos, en torno a ejes específicos que tienen un denominador común; representa una orientación valorativa puesto que las decisiones, la asignación de recursos que conllevan, implica necesariamente orientaciones de valor tanto desde el punto de vista de las grandes metas que se pretenden alcanzar, como las funciones y responsabilidades de los distintos involucrados en la política; implica capacidades legales para usar la coerción, promover acuerdos o usar incentivos; tiene costos, toda política implica costos en un contexto de escasez de recursos.

Una de las mayores dificultades que se encuentran durante el estudio de las políticas públicas está asociada al apuro que tienen los analistas para delimitar el objeto de estudio y diferenciarlo de otras acciones gubernamentales que se pueden dar sobre los problemas sociales; así mismo, existen dificultades de distinción entre lo que es una política pública y otro tipo de estrategias que se dan desde el gobierno con el propósito de reorganizar o administrar el Estado y mejorar la gobernabilidad de lo público (Torres-Melo \& Santander, 2013).

Formalmente, la investigación sobre las políticas públicas surge y crece bajo el enfoque positivista con la ambición de fundar una ciencia de la política pública, sobre todo, en Estados Unidos después de la Segunda Guerra Mundial, donde el libro “The Policy Sciences” de Lasswell \& Lerner, constituye la primicia del planteamiento que invita a indagar el papel del conocimiento y racionalidad en el proceso de elaboración de las políticas públicas (Cruz \& Mballa, 2017). 
Una política pública no es cualquier acción del gobierno, que puede ser singular y pasajera, en respuesta a particulares circunstancias políticas o demandas sociales; lo específico y peculiar de la política pública consiste en ser un conjunto de acciones intencionales y causales, orientadas a la realización de un objetivo de interés / beneficio público, cuyos lineamientos de acción, agentes, instrumentos, procedimientos y recursos se reproducen en el tiempo de manera constante y coherente (con las correcciones marginales necesarias), en correspondencia con el cumplimientos de funciones públicas que son de naturaleza permanente o con la atención de problemas públicos, cuya solución implica una acción sostenida; la estructura estable de sus acciones, que se reproduce durante un cierto tiempo, es lo esencial y específico de ese conjunto de acciones de gobierno que llamamos política pública (Aguilar \& Lima, 2009).

La política pública implica muchos actores trabajando sobre temáticas complejas, que no siempre son sectoriales; donde existen relaciones entre la educación, salud, aseguramiento universal, aseguramiento de la desnutrición en lo global, entre otros (Salazar, 2007).

Una política pública es un proceso integrador de decisiones, acciones, inacciones, acuerdos e instrumentos, adelantado por autoridades públicas con la participación eventual de los particulares, y encaminado a solucionar o prevenir una situación definida como problemática; la política pública hace parte de un ambiente determinado del cual se nutre y al cual pretende modificar o mantener (Velásquez, 2009).

\section{Sobre diseño de políticas públicas}

La construcción de políticas públicas, es una actividad compleja que requiere fortalezas intelectuales y empíricas, implica articular la toma de decisiones, ser sujetos receptores de impacto, requiere formulación democrática que fortalezca la ciudadanía, implica formular objetivos claros que permitan ser evaluados y monitoreados permanentemente, implica un proceso de gestión transparente (Salazar, 2007); no es una estrategia de moda que pueda ser abordada con improvisación por parte de los actores políticos, de ahí la importancia de la academia y el rol de la contraloría social en este nuevo escenario público.

La existencia de una política pública plantea la presencia de una estructura que ordena la acción del Estado; su decisión debe verse reflejada en un ordenado conjunto de acciones, agregadas en distintos 
niveles de complejidad; una política pública puede componerse de cuatro niveles asociados: (i) el estratégico, (ii) el de planeación, (iii) el de programación y (iv) el de acciones de política (TorresMelo \& Santander, 2013).

De acuerdo con Olavarría (2007), toda política pública presenta cuatro elementos. Primero, que para que haya una política pública debe haber una decisión o conjunto de decisiones sobre un mismo tema, de una autoridad competente; segundo, que esa decisión o conjunto de decisiones siguen un proceso formal, un procedimiento institucional que lleva a que ciertas iniciativas se transformen o no en una política pública; tercero, la caracterización del problema y la discusión sobre los aspectos sustantivos de la política pública hace necesario el aporte de la aproximación técnico - racional; y cuarto, que tras la definición de una política pública hay un proceso político en que diversos actores explicitan sus intereses y pugnan porque los contenidos concretos de la política pública incorpore y satisfaga esos intereses.

Existen coincidencias generales en señalar dentro del proceso de políticas públicas, cinco momentos básicos (que pueden no explicitarse o acontecer en forma lineal) (Lupi, Rossi \& Mafullo, 2014); a saber, 1) Identificación de un problema, 2) Formulación de una acción, 3) Toma de decisión por parte del Estado y actores relevantes, 4) Aplicación de la acción, y 5) Evaluación de los resultados.

El diseño de las propuestas de políticas públicas y la elección de los instrumentos que se utilizarán para materializar la intervención es una tarea que reviste características de trabajo técnico, dentro del proceso de la política pública. El diseño de las propuestas es un trabajo que requiere comprender y caracterizar el problema, definir las propuestas de intervención y seleccionar los instrumentos que se usarán para materializar la política pública. En el proceso técnico del diseño, el analista deberá acopiar información que le permita comprender de qué se trata el problema, su tamaño y cuáles son sus alcances. El diseño de las propuestas involucrará tanto la identificación de cursos de acción que permitan resolver o aminorar el problema público, como también delinear la estrategia política que permita aprobar la propuesta. En el diseño de las propuestas los analistas enfrentaran restricciones sustantivas, institucionales o políticas. Adicionalmente, dado que las alternativas entrarán a un proceso político de selección y descarte, para determinar la fortaleza de sus propuestas los analistas evaluarán los resultados de acuerdo a criterios comúnmente utilizados, como efectividad y eficiencia, criterios valóricos y criterios prácticos referidos a la legalidad, aceptación política y aplicabilidad de 
la propuesta. En el diseño de la propuesta el analista aplicará los instrumentos que están a su disposición. Entre ellos: gestión directa del gobierno, privatizaciones, regulaciones, mecanismos de mercado, impuestos, subsidios, campañas de educación, información y persuasión (Olavarría, 2007).

El ambiente de la política pública está conformado por diversos elementos; entre ellos, el sistema político dentro del cual se forma y que está integrado por el ordenamiento jurídico, por las organizaciones públicas y por las comunidades que se benefician o se ven afectadas por la política misma. De este sistema político, en particular de su ordenamiento jurídico, las autoridades públicas derivan su poder para participar en el proceso de formación de la política. Por ello, un concepto completo de política pública tiene que integrar al ambiente como elemento esencial (Velásquez, 2009).

La capacidad para diseñar políticas públicas está vinculada a aspectos fundamentales como la coordinación y los procesos de participación, flexibilidad para orientar y concertar las prioridades, innovación para realizar las transformaciones pertinentes, calidad para distinguir lo que es sustantivo (estratégico) en el proceso de transformación social deseada que se busca alcanzar, considerando las diversas fases de formulación- implementación y evaluación (sostenibilidad social-económica y ambiental) por medio de rendición de cuentas, seguimiento y análisis de resultados y la legitimidad en todo el ciclo de la política (Mideplan, 2016).

\section{Otros aspectos clave en el diseño de políticas públicas, incluye su análisis y evaluación}

El análisis de política pública es un campo de trabajo profesional práctico, orientado a la solución de problemas y multidisciplinario. El análisis de política pública, se hace para comprender las situaciones que han sido, o están en vías de ser identificadas como problemas públicos, para asesorar a las autoridades y otros actores claves en el proceso de la política pública, para apoyar y promover la participación ciudadana en la discusión de problemas y políticas públicas, y para influir en su diseño e implementación. Asimismo, el análisis de política pública permite describir la situación que está siendo abordada por una política pública, establecer si hay correspondencia entre el problema identificado y la política que busca resolverlo o aminorarlo, e identificar las consecuencias de la implementación de la política pública en la forma de resultados, impactos y si fue efectiva, eficaz y/o eficiente. Finalmente, el analista de política pública también deberá considerar una estrategia política 
que le otorgue razonables posibilidades de éxito que la iniciativa será aprobada e implementada (Olavarría, 2007).

La evaluación de las políticas debe hacerse con referencia a los objetivos establecidos, pero sobre la base fundamental de los resultados alcanzados, tanto en los aspectos previstos como en los no previstos; en su forma más simple y esquemática, la evaluación consistiría, pues, en el proceso necesario para medir el grado en el que se están alcanzando las finalidades deseadas y sugerir los cambios que puedan situar las realizaciones de la política más en la línea esperada (Pallares, 1988).

\section{Participación ciudadana en las políticas públicas}

El tema de la participación ciudadana en los procesos de políticas públicas se considera importante, porque constituye un elemento fundamental y condición de posibilidad para la gobernanza democrática, y puede llegar a constituir un mecanismo para el empoderamiento social (Villarreal, 2009).

La participación ciudadana es el proceso de la inclusión de la ciudadanía en la toma de decisiones públicas de diversa índole que afecten o influyan algún aspecto de su vida, con el fin de mantener o no el orden establecido (Castillo, 2017). La participación ciudadana es el involucramiento de los individuos en el espacio público estatal y no estatal desde su identidad de ciudadanos, es decir, de integrantes de una comunidad política, en donde dicha participación está vinculada a los modelos de democracia y al tipo de relación gobierno-sociedad que se quiere construir (Villarreal, 2009).

Las políticas públicas son un conjunto de decisiones y acciones realizadas por una autoridad pública o gobierno que están dirigidas a alcanzar objetivos públicos, solucionar o por lo menos disminuir un problema o necesidad que se presenta como insatisfactorio por la ciudadanía. Las políticas públicas son respuestas concretas a la pregunta acerca de cuándo y cómo debe intervenir el Estado en la sociedad (Castillo, 2017).

Al relacionar la participación ciudadana con las políticas públicas, se evidencia una estrecha relación entre ambos conceptos, pues la participación ciudadana se define como un elemento esencial para influenciar las decisiones políticas, mientras que las políticas públicas son respuestas a demandas elaboradas por la ciudadanía: hay correlación directa entre ambas (Castillo, 2017). 
La participación ciudadana con carácter político busca intervenir en la determinación de los fines, los valores y los intereses sociales apelando a la racionalidad comunicativa mediante la presentación de argumentos racionales (Villarreal, 2009).

\section{Políticas públicas en el contexto universitario}

Los mecanismos de participación ciudadana están destinados para reforzar el constante diálogo y la capacidad de reflexión de los ciudadanos comunes en materia de asuntos públicos, creando mayor cultura política; estos mecanismos deben incentivar la participación de nuevos actores distintos a los tradicionales en la formulación de políticas y decisiones públicas (Cruz \& Mballa, 2017).

Un espacio de la sociedad que está incrementando la participación de la ciudadanía es la Universidad; instituciones que habían permanecido reservadas para algunos actores de la sociedad, y que ahora, con el conocimiento del ciudadano común de que las mismas son gestionadas con recursos del Estado, sienten que tienen derecho al uso de sus servicios académicos y no académicos.

Como lo señala Acosta (2013), en términos de la educación superior universitaria, los contextos institucionales han experimentado transformaciones importantes en las últimas dos décadas, debido en parte a los cambios en los paradigmas de políticas educativas que se han instrumentado. Agrega Acosta que, en estos cambios contextuales, las políticas públicas y las políticas institucionales operan como mecanismos directos o indirectos de formación de incentivos y recompensas para la formulación de las decisiones individuales, pero también introducen nuevas zonas de incertidumbre para los individuos y para las instituciones.

Por otro lado, las políticas públicas universitarias se han reorientado a la identificación de oportunidades de autofinanciamiento; esto, dada la cuestión de escasez relativa de recursos por parte de las finanzas públicas, las cuales han convertido el proceso de formulación de políticas públicas en un tema clave para la gestión universitaria.

Las políticas públicas, definidas por la Secretaría Nacional de Planificación y Desarrollo (SENPLADES, 2011) como un curso de acción de la gestión pública que institucionaliza la intervención pública en respuesta a un problema social identificado como prioritario, y que se convierte de esta manera en materia de política de Estado, forman parte del quehacer universitario. 
La transformación de la educación en espacio público, y por ende su gestión, la hace idónea para la intervención de las políticas públicas y de las autoridades públicas, a través de un conjunto de iniciativas e instrumentos; esto que aparece como una característica propia de una sociedad moderna, es el resultado de procesos sociales complejos y de luchas sociales y políticas (Arcos, 2008).

Por otra parte, dado que la universidad se encuentra inmersa en la sociedad, en relación dinámica con ésta, en un diálogo permanente y reciproco, cabe señalar que ésta cumple una función social; en consecuencia se sostiene que la actividad universitaria no puede centrarse exclusivamente a la práctica académica, sino que la gestión socialmente responsable de la universidad se traduce en una suma de esfuerzos colectivos, que implica la gestión de organización misma, de la formación académica, de la producción del saber y de la participación, orientada al desarrollo humano sostenible, en virtud de lo cual involucra diferentes actores sociales y matices tanto económicos, sociales, éticos y filosóficos (Castañeda et al, 2007).

Como lo señalan Osorio, Blanco \& Rositas (2013), para tener acceso a los recursos que la educación superior requiere, las Instituciones de Educación Superior (IES) y sus académicos se ven sujetos al cumplimiento de una serie de parámetros de "calidad" establecidos; agregan que, para algunos académicos es claro el vínculo entre educación y condiciones materiales de vida, por lo que privilegian la importancia de la universidad como el espacio donde se produce y se distribuye el conocimiento, mientras que otros perciben una contradicción del propio quehacer de la universidad y la de ellos mismos como sujetos.

Por su parte Acosta (2013), plantea dos tipos de políticas en el contexto universitario; las generadas por la propia la universidad- y las que se derivan del nuevo entorno de políticas públicas de la educación superior, que suponen una suerte de esfuerzos de "adaptación incremental" de las universidades a las exigencias y reglas contendidas en dichas políticas, expresadas empíricamente en los diversos programas gubernamentales que involucran recursos extraordinarios, reconocimientos o prestigios a las propias universidades, a los grupos académicos (cuerpos académicos) o a los individuos (profesorado y estudiantes).

La mayoría de las investigaciones en el contexto universitario, le dan un enfoque hacia la investigación, y no hacía el diseño de políticas publican dentro del contexto universitario. Trabajos 
como los de Capera \& Galeano (2017), realizan un abordaje teórico, conceptual y metodológico referente a un acercamiento de la literatura de las políticas públicas latinoamericanas, y planteando su importancia como objeto de estudio para la ciencia política y la administración pública.

En cuanto al contex to ecuatoriano, Toscanini, Aguilar \& García (2016) señalan que, el Ecuador cuenta con la Ley Orgánica de Educación Superior, coherente con la Constitución de la República, con reglamentos, normas y resoluciones administrativas que armonizan el desempeño de las IES, a partir del liderazgo de los organismos competentes y responsables de este proceso en el país. Agregan que, las actuales políticas estatales han impuesto un riguroso accionar y manejo de las IES, bajo condicionamientos estrictos, con miras al mejoramiento individual y colectivo, y destacando el estímulo a la investigación, a la mejora académica, así como a una adecuada selección del recurso humano estudiantil.

Las políticas públicas nacionales inciden de forma definitiva en los resultados de producción y publicación científica de los países latinoamericanos; estos países, en particular Ecuador, están en un momento de desarrollo y adaptación a los esquemas de evaluación y calidad que existen en los países desarrollados; las condiciones políticas y económicas de la región latinoamericana han sido favorables en muchos aspectos en el último lustro, aunque pocos de estos países han aprovechado este momento (Álvarez-Muñoz \& Pérez-Montoro, 2016).

Como parte de las políticas públicas y a propósito de los excedentes petroleros, en el Ecuador se transfirieron gran parte de estos recursos a procesos de mejora de sus respectivos sistemas de educación superior, creando políticas de inversión que han permitido dar saltos cualitativos y cuantitativos en sus procesos de producción, comunicación y visibilidad científica (Álvarez-Muñoz \& Pérez-Montoro, 2016).

Otro factor presente en el debate de la participación de políticas públicas en el contexto universitario es la interculturalidad; la cual según Cruz (2015), ha tomado presencia en Ecuador, como en otros países de América Latina, convirtiéndose en objeto de múltiples y controvertidas discusiones en torno a su concepción, y está sirviendo como paradigma de las políticas públicas del Estado, especialmente en materia de educación, salud y cultura.

\section{Reflexiones finales}


Se debe trabajar más en la institucionalización académica e investigativa del estudio de las políticas públicas, para ello, se debe asumir un trabajo introspectivo que mire hacia al pasado con el fin de detectar y comprender la forma como el estudio de las políticas ha venido evolucionado y así, asumir el trabajo prospectivo de reflexionar sobre sus posibilidades actuales y futuras para afrontar las realidades problemáticas de las distintas sociedades (Valencia \& Álvarez, 2008).

Las políticas públicas son un campo de investigación que tiene múltiples posibilidades al interior de la administración pública y la ciencia política, para generar procesos que tengan como referente las teorías, las acciones y los modelos de aplicación en la sociedad civil; en esa perspectiva, queda la ardua tarea de seguir avanzando en función de construir resultados que tengan en cuenta elementos económicos, políticos, administrativos, organizacionales, conductuales y de amplia planificación en las prácticas, que dan viabilidad a los proyectos de intervención social, estos encargados de materializar la formulación y aplicación de una política pública, un programa social o un proyecto político al interior de la sociedad civil y las instituciones públicas latinoamericanas (Capera \& Galeano, 2017).

A su vez, debe ser un foco que aclare y oriente la gestión pública de los gobernantes, quienes, en ocasiones, asumen medidas inmediatas, oportunistas e ineficientes; las cuales hacen más daño que bien a la sociedad, así como a la legitimidad y funcionalidad del aparato estatal. En su relación con la sociedad civil, las políticas públicas se constituyen en un poderoso instrumento de comunicación con el Estado y una buena forma como los gobernantes podrían cambiar el rumbo de manera positiva a las condiciones de pobreza y marginalidad actual (Valencia \& Álvarez, 2008).

La construcción de una gobernanza democrática demanda la participación de ciudadanos dialogantes y solidarios, más que individuos ocupados en maximizar su propio interés; urge, por tanto, ampliar la reflexión sobre las mejores herramientas institucionales que impulsen la intervención a la vez crítica, vigilante y cooperadora de los ciudadanos en la gestión pública, para orientarla hacia el bienestar colectivo (Villarreal, 2009).

\section{Referencias Bibliográficas}

Acosta, A. (2013). Políticas, actores y decisiones en las universidades públicas en México: un enfoque institucional. Revista de la educación superior, 42(165), 83-100 
Aguilar, C. \& Lima, M. (2009). ¿Qué son y para qué sirven las políticas públicas? Contribuciones a las Ciencias Sociales, EUMED

Álvarez-Muñoz, P. \& Pérez-Montoro, M. (2016). Políticas científicas públicas en Latinoamérica: el caso de Ecuador y Colombia. El profesional de la información, 25(5), 758-766. DOI: https://doi.org/10.3145/epi.2016.sep.06

Arcos, C. (2008). Política pública y reforma educativa en el Ecuador. En “Desafíos para la educación en el Ecuador: calidad y equidad”, Arcos, C. \& Espinosa, B. (coord.), 29-63. Quito: FLACSO

Bromell, D. (2012). Doing the right thing: Ethical dilemmas in public policy making. Centre for Theology and Public Issues. Working Paper. New Zealand: University of Otago

Capera \& Galeano (2017). Las políticas públicas un campo de reflexión analítica entre la ciencia política y la administración pública latinoamericana. TELOS, 19(2), 343-365

Castañeda, G.; Ruiz, M.; Viloria, O.; Castañeda, R. \& Quevedo, Y. (2007). El rol de las universidades en el contexto de la responsabilidad social empresarial. Negotium, 3 (8), 100-132

Castillo, M. (2017). El papel de la participación ciudadana en las políticas públicas, bajo el actual escenario de la gobernanza: reflexiones teóricas. Revista CS, 23, 157-180. DOI: http://dx.doi.org/10.18046/recs.i23.2281

Cruz, (2015). ¿Interculturalidad para todos? Políticas públicas y prácticas educativas en Ecuador. Perifèria, 20 (1), 110-148. DOI: https://doi.org/10.5565/rev/periferia.457

Cruz, L. \& Mballa, L. (2017). Mecanismos de participación ciudadana en las políticas públicas en América Latina. Revista Políticas Públicas, 10(1), 1-25

Lahera, E. (2004). Política y políticas públicas. Serie políticas sociales. Santiago de Chile: CEPAL

Lupi, C.; Rossi, D. \& Mafullo, E. (2014). Estado, políticas públicas y comunicación. Buenos Aires: Universidad de Buenos Aires 
Ministerio de planificación nacional y política económica (2016). Guía para la elaboración de políticas públicas. San José, Costa Rica: Mideplan

Olavarría, M. (2007). Conceptos básicos en el análisis de políticas públicas. Documentos de trabajo, 11, Instituto de Asuntos Públicos, Universidad de Chile

Osorio, J.; Blanco, M. \& Rositas, J. (2013). Políticas públicas en educación superior su impacto en las universidades públicas. Primer Congreso Internacional de Investigación Educativa RIE-UANL. Recuperado de: http://eprints.uanl.mx/8151/1/o2_1.pdf

Pallares, F. (1988). Las políticas públicas: el sistema político en acción. Revista de estudios políticos, $62,141-162$.

Salazar, P. (2007). Políticas públicas sociales para pueblos indígenas. En "Políticas públicas para pueblos indígenas en el ecuador del siglo xxi. Memorias del seminario nacional”. Quito: Escuela de Gobierno y Políticas Públicas / Fundación Konrad Adenauer

Secretaría nacional de planificación y desarrollo (2011). Guía para la formulación de políticas públicas sectoriales. Quito: Senplades

Torres-Melo, J. \& Santander, J. (2013). Introducción a las políticas públicas: conceptos y herramientas desde la relación entre Estado y ciudadanía. Bogotá: Instituto de Estudios del Ministerio PúblicoIEMP

Toscanini, M.; Aguilar, A. \& García, R. (2016). Diagnóstico de las políticas públicas de la educación superior en el Ecuador. Revista Cubana de Educación Superior, 3, 161-178

Valencia, G. \& Álvarez, Y. (2008). La ciencia política y las políticas públicas: notas para una reconstrucción histórica de su relación. Estudios políticos, 33, 93-121

Velásquez, R. (2009). Hacia una nueva definición del concepto “política pública”. Desafíos, (20), $149-187$

Villarreal, M. (2009). Participación ciudadana y políticas públicas. Décimo certamen de ensayo político, 31-48 